\title{
Presentation of Acute Lymphoblastic Lymphoma and Colorectal Carcinoma in the Context of Constitutional Mismatch Repair Deficiency Syndrome: A Case Report with Literature Review
}

\author{
Muhammad Irfan Basheer ${ }^{1}$, Iftikhar Ali Rana ${ }^{1}$, Umer Nisar Sheikh ${ }^{1}$, \\ Muhammed Aasim Yusuf ${ }^{2}$, Irfana Ishaq Sindhu ${ }^{3}$, Asif Loya ${ }^{1}$ \\ 1Department of Pathology, Shaukat Khanum Memorial Cancer Hospital and Research Centre, Lahore, \\ Pakistan, ${ }^{2}$ Department of Internal Medicine, Shaukat Khanum Memorial Cancer Hospital and Research \\ Centre, Lahore, Pakistan, ${ }^{3}$ Department of Paediatric Oncology, Shaukat Khanum Memorial Cancer \\ Hospital and Research Centre, Lahore, Pakistan
}

Received: 28 November 2021/Accepted: 20 December 2021

\section{Open ACCESS}

Correspondence:

Muhammad Irfan Basheer, 7A Block R-3, Phase 2, M.A. Johar Town, Lahore, Punjab, 54782 Pakistan.

E-mail: dr.irfanbasheer@gmail.com

Citation: Basheer MI, Rana IA, Sheikh UN, Yusuf MA, Sindhu II, Loya A. Presentation of Acute Lymphoblastic Lymphoma and Colorectal Carcinoma in the Context of Constitutional Mismatch Repair Deficiency Syndrome: A Case Report with Literature Review. $\mathrm{J}$ Cancer Allied Spec [Internet]. 2022 Jan 31;8(1). XXX.

https://doi.org/110.37029/jcas. v8i1.443

Copyright: () 2022 Basheer, et al.This is an open access article distributed under the terms of the Creative Commons Attribution License, which permits unrestricted use, distribution, and reproduction in any medium, provided the original author and source are credited.

Funding: This research received no specific grant from any funding agency in the public, commercial or not-for-profit sectors.

Competing interest: Nil.

\begin{abstract}
Introduction: Constitutional mismatch repair deficiency (CMMRD) is a rare autosomal recessive disease carrying an increased risk of cancers (paediatric tumours of central nervous system, haematolymphoid malignancies along with gastrointestinal (GI) cancer(s), which are usually seen in the second and third decades), leading to syndromic presentation. Causal mutations are detected in DNA mismatch repair (MMR) genes, including MLH1, PMS2, MSH2 and MSH6 that are also known for their established role in Lynch syndrome. We describe a case of CMMRD with an earlier (first decade of life) presentation of mediastinal acute lymphoblastic lymphoma and colorectal malignancy. Case Presentation: A 5-year-old boy presented with respiratory complaints, bilateral cervical lymphadenopathy, multiple café-au-lait macules (CALMs) on the lower back and history of parental consanguinity with the death of three sisters due to brain tumour within 6 months of diagnosis. Computerised tomographic scan chest revealed a huge mediastinal mass. The patient underwent a trucut biopsy of the mass. The results were significant for a pre-T-cell acute lymphoblastic lymphoma. Suspicion of CMMRD was raised based on a combination of factors described above. A panel of MMR proteins was applied on the biopsy tissue that revealed loss of nuclear expression of MLH1 and PMS2 immunostaining in tumour cells with positive external controls. While on maintenance therapy for lymphoma, about a year later, the patient developed subacute intestinal obstruction due to a stenosing polypoidal circumferential tumour in the mid-sigmoid colon found on flexible sigmoidoscopy that was followed by endoscopic biopsies and insertion of a fully covered self-expanding metallic adult biliary stent with a diameter of $10 \mathrm{~mm}$ and length of $6 \mathrm{~cm}$ leading to immediate relief of obstruction. Biopsies revealed adenocarcinoma with neuroendocrine differentiation. Metastatic tumour deposits were seen in the omentum, anterior abdominal wall and the left peritoneal wall. Practical Implications: Earlier (first decade) presentation of GI malignancy warrants that an earlier screening
\end{abstract}


through radiological scans for any possible tumours and MMR protein expression analysis (loss in tumour plus normal non-tumour cells) are essential in patients having CALMs and family history of paediatric tumours.

Key words: Café-au-lait macules, constitutional mismatch repair deficiency, gastrointestinal tract, mismatch repair proteins

\section{Introduction}

Constitutional mismatch repair deficiency (CMMRD) is a rare condition carrying an increased risk of cancers, leading to a syndromic presentation. ${ }^{[1]}$ Causal mutations are detected in DNA mismatch repair (MMR) genes, including MLH1, PMS2, MSH2 and MSH6, which are also known for the established role in the causation of Lynch syndrome. ${ }^{[2,3]}$ CMMRD has an autosomal recessive inheritance pattern and presents with paediatric tumours of the central nervous system (CNS) and haematolymphoid malignancies along with gastrointestinal (GI) cancer(s), which are usually seen in the second and third decades of life of a patient affected by CMMRD. There is a high tendency of multiple (synchronous and metachronous) cancers associated with a poor prognosis, in general. ${ }^{[4,5]}$ In this case report, we describe a case of CMMRD with a presentation of mediastinal acute lymphoblastic lymphoma and colorectal malignancy, which presented in the first decade of life. This is a very rare and seldom reported occurrence and there are only a few published case reports of colorectal malignancy in the first decade of life in a patient of CMMRD.

\section{Case Presentation}

A 5-year-old boy presented with cough and shortness of breath for 1 month, bilateral cervical lymphadenopathy, multiple café-au-lait macules (CALMs) on the lower back and history of parental consanguinity with the death of three sisters due to brain tumour within 6 months of diagnosis. Pedigree study did not reveal any tumour in the parents and/or other family members. Computerised tomographic (CT) scan chest revealed a huge mediastinal mass with bilateral pleural effusion.

\section{Diagnosis and Management}

A trucut biopsy of the mass was performed. The report was suggestive of a pre-T-cell acute lymphoblastic lymphoma [Figure 1a]. In a weekly multidisciplinary team meeting, suspicion of CMMRD was raised based on a combination of factors, including a family history of CNS tumours, parental consanguinity and haematolymphoid malignancy. Therefore, a panel of microsatellite instability surrogate immunomarkers (MMR proteins) was applied on the tissue biopsy that revealed loss of nuclear expression of MLH1

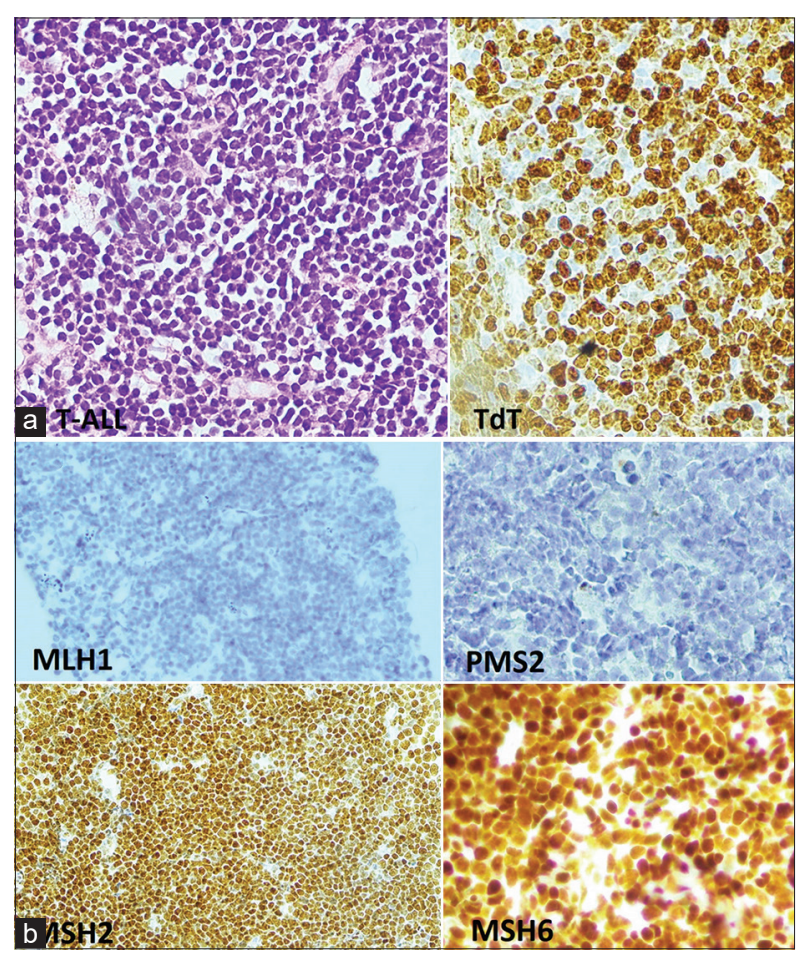

Figure 1: (a) Typical morphology of acute lymphoblastic lymphoma with tumour cells highlighted by TdT immunostain, (b) Loss of MLH1 and PMS2 staining in lymphoblastic lymphoma with retained immunoexpression of $\mathrm{MSH} 2$ and $\mathrm{MSH} 6$ 
and PMS2 proteins in tumour cells with positive external controls [Figure 1b]. Chemotherapy was started according to the United Kingdom Acute Lymphoblastic Lymphoma/Leukaemia Trial interim guidelines 2019 regimen C. On maintenance therapy for lymphoma, the patient developed subacute intestinal obstruction about a year later. A CT scan showed a descending colon stricture/mass, and urgent subsequent flexible sigmoidoscopy showed a stenosing polypoidal circumferential tumour in the mid-sigmoid colon. Endoscopic biopsies were taken, and a fully covered self-expanding metallic stent was inserted, which led to immediate relief of obstruction and rapid resolution of symptoms. Biopsies were reported as showing adenocarcinoma with neuroendocrine differentiation [Figure 2]. The use of a self-expanding metallic stent in a child with malignant bowel obstruction has not previously been reported in the literature. In the absence of specific paediatric stents for use in such a situation, an adult biliary stent, with a diameter of $10 \mathrm{~mm}$ and length of $6 \mathrm{~cm}$ (Lot No. 21030218), was used. It helped avoid emergency surgery with its attendant risks and morbidity. Elective left haemicolectomy was performed 3 weeks later. Metastatic tumour deposits were seen in the omentum, and along the anterior abdominal wall and the left peritoneal wall. A panel of MMR proteins was again applied on this specimen [Figure 3] that confirmed the absence of MLH1 and PMS2 immunostaining both in tumour cells and in normal/benign non-tumour colonic glands. At present, the patient is on maintenance chemotherapy for lymphoma and on follow-up only for the second colonic malignancy. Informed consent was obtained from the parent of the patient for the publication of the case report followed by the approval from the Institutional Review Board of Shaukat Khanum Cancer Hospital and Research Centre.

\section{Discussion}

CMMRD is the term used for a rare familial syndrome first recognised in 1999, and since then, about 200 plus case reports have been reported. ${ }^{[1]}$ The genetic basis for CMMRD lies in biallelic germline mutations in MMR genes. ${ }^{[2,3]}$ A more significant proportion (about 60\%) of CMMRD cases result from a mutation in PMS2 followed by a mutation in MSH6 (20-30\% cases) and mutation in MLH1/ MSH2 genes (10-20\%). ${ }^{[1]}$ Pedigree analysis of the patients affected by CMMRD shows an autosomal recessive inheritance pattern. In CMMRD, children and adolescents are usually affected by tumours of the CNS, GI tract (GIT), haematolymphoid system and Lynch syndrome-related tumours, including carcinomas of endometrial and urinary tract origin.

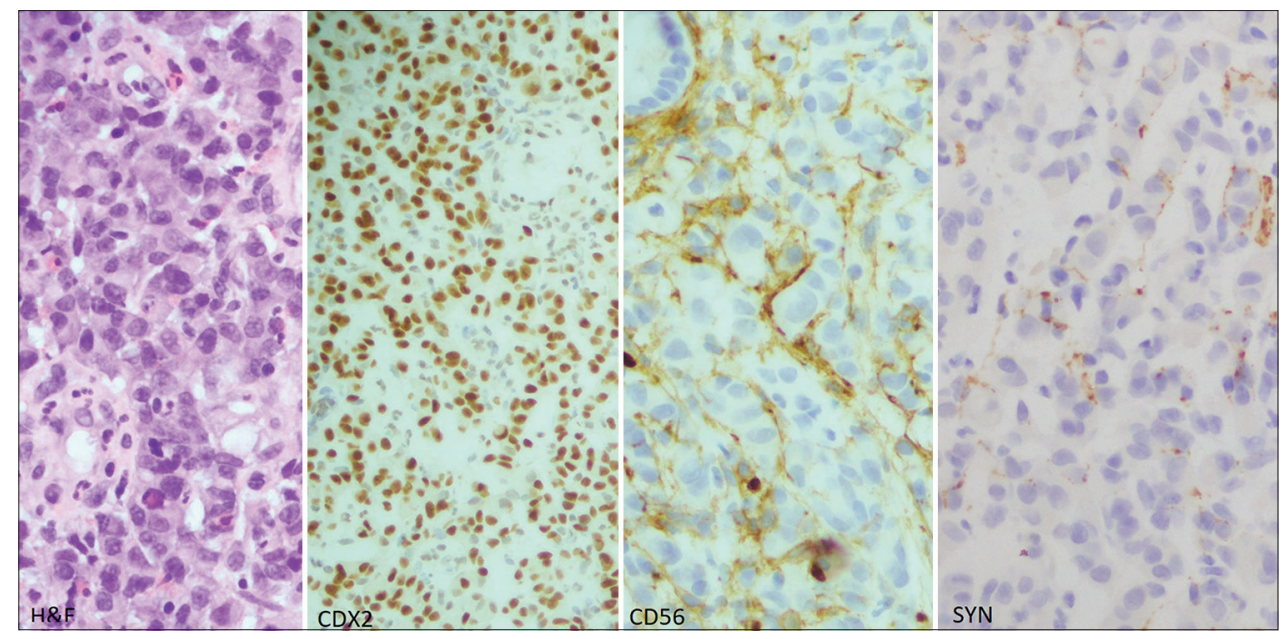

Figure 2: Adenocarcinoma colon with the evidence of intestinal differentiation by CDX2 immunostain and neuroendocrine differentiation by patchy staining of CD56 and synaptophysin immunostain 


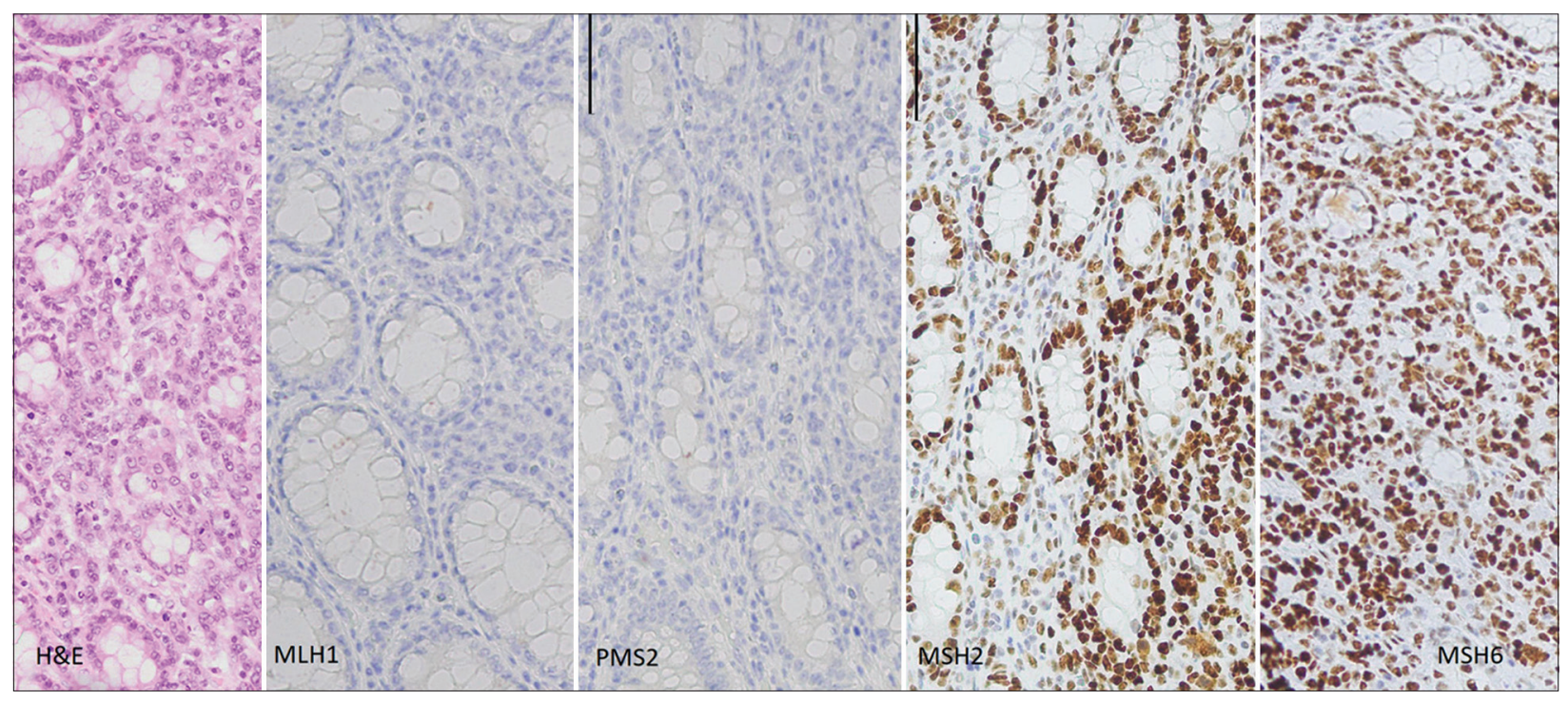

Figure 3: Adenocarcinoma colon (haemicolectomy specimen, panel of mismatch repair proteins applied). Note the absence of MLH1 and PMS2 immunostaining both in tumour cells and intervening normal/benign colonic glands. Note the presence of MSH2 and MSH6 immunostaining both in tumour cells and intervening normal/benign colonic glands

The CNS tumours in CMMRD include high-grade gliomas, medulloblastomas and supratentorial primitive neuroectodermal tumours. Majority of the brain tumours in CMMRD syndrome present before 25 years of age with a mean age of 8 years. ${ }^{[1]}$ The majority of haematolymphoid tumours in CMMRD syndrome are diagnosed before 10 years of age, with the mean age being 5.5 years. ${ }^{[1]}$

Gl tumours in CMMRD are age dependent. These include adenomas with or without high-grade dysplasia and polyps resembling those seen in juvenile polyposis. ${ }^{[6]}$ GIT cancers represent about $37 \%$ of all tumours of CMMRD syndrome..$^{[1]}$ The CMMRD-associated intestinal carcinomas comprise about $2.1 \%$ of all intestinal carcinomas before 35 years. ${ }^{[7]}$ Colorectal tumours (adenomas and carcinomas) predominantly involve the left side of the colon in CMMRD syndrome rather than the right side as seen in Lynch syndrome. ${ }^{[10]}$ Tumours of GIT in CMMRD are usually seen in the second and third decades of life as secondary or tertiary malignancy. ${ }^{[2]}$ However, in this case, GIT malignancy presented earlier in almost the middle of the first decade of life, which is quite unusual and has been presented in three case reports so far. ${ }^{[10-14]}$ CALMs are a helpful clinical finding in diagnosing CMMRD. However, an astute clinical examination may be required in finding CALMs and then differentiating these lesions from the similar spots seen in neurofibromatosis (NF) type 1. However, CALMs seen in CMMRD have a somewhat irregular appearance having variability in degrees of pigmentation compared with those seen in NF type $1 .{ }^{[4,5]}$ Identifying persons having CMMRD syndrome requires a challenging approach in terms of awareness of its clinical features and diagnostic caveats.

Immunohistochemical (IHC) study of MMR proteins is an ancillary test used as a surrogate indicator of mutation of respective genes of MMR proteins identified by loss of expression of one of the proteins (MLH1, PMS2, MSH2 and MSH6). Diagnosis of CMMRD requires loss of at least one of MMR proteins both in tumour cells and in normal tissue of the patient being investigated (positive control from another healthy individual should be included during $\mathrm{IHC}$ procedure). Hence, any unusual immunostaining pattern of MMR protein in neoplastic and normal/ benign non-tumour cells should be taken as a guide toward mutation assessment of MMR genes. ${ }^{[8,9]}$ 
Earlier (first decade) presentation of GIT malignancy warrants that a clinician is more vigilant and does earlier surveillance for CMMRD in the patient as well as other family members. In addition, a peculiarity of CALMs may act as a screening tool for clinicians to suspect CMMRD. Initial workup, including radiological scans for any possible tumours and MMR protein expression analysis (loss in tumour cells plus normal non-tumour cells), is essential in such patients to reach an earlier diagnosis and possible better prognosis.

\section{Acknowledgement}

Muhammad Ishaq for facilitation in IHC work-up of this case.

\section{References}

1. Wimmer K, Kratz CP, Vasen HF, Caron O, Colas C, Entz-Werle N, et al. EU-consortium care for CMMRD (C4CMMRD). Diagnostic criteria for constitutional mismatch repair deficiency syndrome: Suggestions of the European consortium 'care for CMMRD' (C4CMMRD). J Med Genet 2014;51:355-65.

2. Ricciardone MD, Ozçelik T, Cevher B, Ozdag H, Tuncer M, Gürgey A, et al. Human MLH1 deficiency predispones to hematological malignancy and neurofibromatosis type 1. Cancer Res 1999;59:290-3.

3. Wang Q, Lasset C, Desseigne F, Frappaz D, Bergeron C, Navarro C, et al. Neurofibromatosis and early onset of cancers in hMLH1-deficient children. Cancer Res 1999;59:294-7.

4. Wang Q, Montmain G, Ruano E, Upadhyaya M, Dudley S, Liskay RM, et al. Neurofibromatosis Type 1 gene as a mutational target in a mismatch repairdeficient cell type. Hum Genet 2003;112:117-23.

5. Brems H, Chmara M, Sahbatou M, Denayer E, Taniguchi K, Kato R, et al. Germline loss-of-function mutations in SPRED1 cause a neurofibromatosis 1-like phenotype. Nat Genet 2007;39:1120-6.

6. Levi Z, Kariv R, Barnes-Kedar I, Goldberg Y, Half $E$, Morgentern $S$, et al. The gastrointestinal manifestation of constitutional mismatch repair deficiency syndrome: From a single adenoma to polyposis-like phenotype and early onset cancer. Clin Genet 2015;88:474-8.

7. Nguyen A, Bougeard G, Koob M, Chenard MP, Schneider A, Maugard C, et al. MSI detection and its pitfalls in CMMRD syndrome in a family with a bi-allelic LH1 mutation. Fam Cancer 2016;15:571-7.

8. Baas AF, Gabbett M, Rimac M, Kansikas M, Raphael M, Nievelstein RA, et al. Agenesis of the corpus callosum and gray matter heterotopia in three patients with constitucional mismatch repair deficiency syndrome. Eur J Hum Genet 2013;21:55-61.

9. Durno CA, Holter S, Sherman PM, Gallinger S. The gastrointestinal phenotype of germline biallelic mismatch repair gene mutations. Am J Gastroenterol 2010;105:2449-56.

10. Wimmer K, Etzler J. Constitutional mismatch repair deficiency syndrome: Have we so far seen only the tip of an iceberg? Hum Genet 2008;124:105-22.

11. Kratz CP, Holter S, Etzler J, Lauten M, Pollett A, Niemeyer CM, et al. Rhabdomyosarcoma in patients with constitutional mismatch-repair-deficiency syndrome. J Med Genet 2009;46:418-20.

12. Durno C, Aronson M, Bapat B, Cohen Z, Gallinger S. Family history and molecular features of children, adolescents, and young adults with colorectal carcinoma. Gut 2005;54:1146-50.

13. Gallinger S, Aronson M, Shayan K, Ratcliffe EM, Gerstle JT, Parkin PC, et al. Gastrointestinal cancers and neurofibromatosis Type 1 features in children with a germline homozygous MLH1 mutation. Gastroenterology 2004;126:576-85.

14. Hegde MR, Chong B, Blazo ME, Chin LH, Ward PA, Chintagumpala MM, et al. A homozygous mutation in MSH6 causes turcot syndrome. Clin Cancer Res 2005; 11:4689-93.

\section{Authors' Contributions}

Conceived and designed the analysis: MIB, IAR, UNS, IIS and AL. Collected the data: MIB, IAR, UNS and IIS. Contributed data or analysis tools: MIB. Performed the analysis: MIB, MAY and AL. Wrote the paper: MIB, IAR, UNS, IIS, MAY and AL. 